\title{
The Dwarf Low Surface Brightness Population in Different Environments of the Local Universe
}

\author{
S. Sabatini ${ }^{1} \dagger$, J. I. Davies ${ }^{2}$, S. Roberts ${ }^{2}$, R. Scaramella ${ }^{1}$ \\ ${ }^{1}$ INAF-Osservatorio Astronomico di Roma, via Frascati 33, 00040 Monte Porzio Catone, \\ Roma,Italy \\ email: sabatini@oa-roma.inaf.it; kosmobob@oa-roma.inaf.it \\ ${ }^{2}$ Department of Physics and Astronomy, Cardiff University, 5 The Parade, \\ Cardiff, CF24 3AA, UK \\ email: Jonathan.Davies@astro.cf.ac.uk; Sarah.Roberts@faulkes-telescope.com
}

\begin{abstract}
The nature of the dwarf galaxy population as a function of location in the cluster and within different environments is investigated. We have previously described the results of a search for low surface brightness objects in data drawn from an East-West strip of the Virgo cluster (Sabatini et al (2003)) and have compared this to a large area strip outside of the cluster (Roberts et al (2004)). In this talk I compare the East-West data (sampling subcluster A and outward) to new data along a North-South cluster strip that samples a different region (part of sub-cluster $\mathrm{A}$, and the N,M clouds) and with data obtained for the Ursa Major cluster and fields around the spiral galaxy M101. The sample of dwarf galaxies in different environments is obtained from uniform datasets that reach central surface brightness values of $26 \mathrm{~B} \mathrm{mag} / \operatorname{arcsec}^{2}$ and an apparent B magnitude of $21\left(\mathrm{M}_{B}=-10\right.$ for a Virgo Cluster distance of $16 \mathrm{Mpc}$ ). We discuss and interpret our results on the properties and distribution of dwarf low surface brightness galaxies in the context of variuos physical processes that are thought to act on galaxies as they form and evolve.
\end{abstract}

Keywords. dwarf galaxies, low suface brightness galaxies, clusters

\section{Introduction}

Although dwarf galaxies are the most abundant galaxies of the Local Universe, their number counts in the field (measured by the faint end part of the Luminosity Function, LF, Blanton et al (2001), Norberg et al (2002)) are well below those predicted by the Mass Function (MF) of $\Lambda$ CDM hierarchical galaxy formation models (Kauffman, White \& Guiderdoni (1993),Klypin et al. (1999),Moore et al (1999)) - this is known as the 'missing satellites' problem. However, recent discoveries of ultra-faint dwarfs of the Local Group made with sloan data (Irwin et al (2007) and references therein) have doubled the number of known dwarf satellites of the MW, accounting for part of the discrepancy between observations and predictions, even if a factor of 4 is still missing in order to solve the missing satellites problem (Simon \& Geha (2007)). These results prove that, even in the Local Universe, the census of the dwarf galaxy population is still incomplete and the slope $(\alpha)$ of the faint end part of the LF is therefore still subject to revision: selection effects due to the intrinsic faint total magnitude and low surface brightness of dwarf galaxies, have made them particularly elusive in surveys that were not purposely built to select them. On the observational side, the issue is further clouded by non-uniform datasets: different surveys reach different magnitude and surface brightness limits and

$\dagger$ Present address: INAF-OAR, via Frascati 33, Monte Porzio Catone, Roma,Italy 
detection methods and selection criteria vary in the way they identify dwarf galaxies. This can lead to studies of the LF in which different authors report extremely different results even for the same region of sky (e.g. see the Fornax cluster where an extremely steep LF $(\alpha \sim-2.0)$ is found by Kambas et al (2000), whilts a flat one $(\alpha \sim-1.1)$ is reported by Mieske et al, this conf.). In this talk we report on our ongoing work to try and quantify the numbers of Low Surface Brightness (LSB) dwarf galaxies as a function of the environment in the Local Universe (within $20 \mathrm{Mpc}$ ) and to assess and compare their properties. We reduced as much as possible the observational biases with the uniformity of our data and by using exactly the same detection algorithm and selection criteria for identifying dwarf galaxies in all the different environments that we analysed. To reach this goal we put very strong requirements on the way we carried out our work:

- the dataset and the data analysis need to be homogeneuos (as mentioned, data in the literature are highly inhomogeneous, often the detection methods, candidate selection and the resulting completeness of the compared samples are different) - our data are obtained in exaclty the same way for the different environments (same observing instrumentation and setup, same data analysis, detection and selection method);

- data need to go deep both in magnitude and surface brightness (flux limited surveys sample only our neighborhoods for the dwarfs contribution and easily miss further away denser environments like the Virgo Cluster; surface brightness limited surveys miss the contribution by LSB galaxies - e.g. SDSS catalogues miss $50 \%$ of galaxies from $\mu_{50, r} \simeq$ $23.5 \mathrm{mag} / \operatorname{arcsec}^{2}$, Blanton et al (2005)) - our method allows the detection of galaxies with intrinsic properties of $23 \leqslant \mu_{o} \leqslant 26 \mathrm{~B}$ mag/ $\operatorname{arcsec}^{2}$ and $-10 \geqslant M_{B} \geqslant-14$, where $\mu_{o}$ and $M_{B}$ are the $\mathrm{B}$ band exponential central surface brightness and absolute magnitude respectively ;

- data need to uniformly analyse different environments: inhabiting the shallowest potential wells and thus being the most sensitive to internal and external physical processes that control galaxy formation, dwarfs are the best test-beds to investigate how the environment affects galaxy evolution and to estimate the contribution of different physiscal processes (photo-ionization, tidal interactions, SN winds, ram pressure stripping, harassment) - we obtained data in environments of the Local Unieverse of different density (the field, M101, the Ursa Major Cluster, the Virgo Cluster). Given the magnitude limits of our survey (see previous point), the galaxy that we analyse are fainter than those typically detected in the comprehensive survey of the Virgo cluster carried out by Binggeli et al (1984) and extends down towards the properties of the 'classical' dSph galaxies of the Local Group (excluding the recently discovered ultra-faint dwarfs).

\section{Data and data analysis}

The optical data were obtained using the Wide Field Camera (WFC) on the Isaac Newton Telescope, La Palma, Canary Islands as part of the Wide Field Survey (WFS), a multicolour data survey covering over $200 \mathrm{deg}^{2}$ of sky. The WFC is a mosaic of four thinned EEV $4 \mathrm{~K} \times 2 \mathrm{~K}$ CCDs with a pixel size of 0.33 arc sec and total sky coverage of 0.29 $\mathrm{deg}^{2}$. Images on CCD 3 were not used due to its vignetting, this reduced our total field of view to $0.21 \mathrm{deg}^{2}$. The Virgo data, taken during observing runs in 1999 and 2002, consists of two perpendicular strips extending from the centre of the Virgo cluster (defined as M87) outwards by $7 \mathrm{deg}$ and covering a total area of about $28 \mathrm{deg}^{2}$. These 2 data strips sample different regions of the Virgo cluster, with one lying roughly perpendicular to the super-galactic plane (E-W) and covering sub-cluster A and outward, and the other (N-S) almost parallel to it, sampling clouds N,M. The Ursa Major data was obtained in 2002: they consist of 8 fields going along a N-S, E-W cross in the cluster and totalling $1.68 \mathrm{deg}^{2}$. The M101 data was obtained in 2004 and consist of 72 frames covering an area of just 


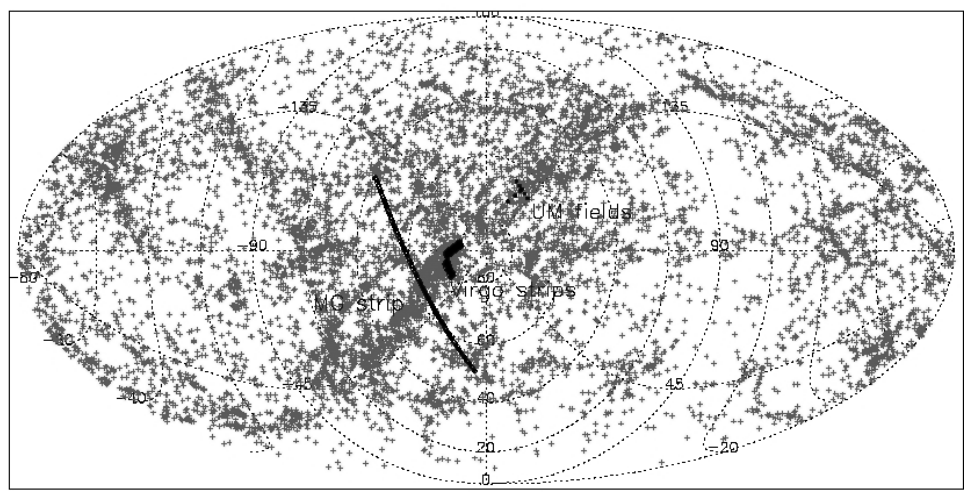

Figure 1. Position of Virgo Cluster data strips, Ursa Major and MGS data viewd from the North galactic pole. Also plotted are all galaxies listed in NED with vi $4500 \mathrm{~km} / \mathrm{s}$.

over $15 \mathrm{deg}^{2}$, in a box around M101. The field region is taken from the Millennium Galaxy Strip (MGS) and consists of an area of $30 \mathrm{deg}^{2}$ along the celestial equator (Liske et al (2003), passing through filaments and voids and crossing the Virgo Southern extension at its mid point. The different environments of this survey are shown in black in Fig 1. All B band exposures were for 750s, whilst the exposure time for the i band images was 1000s. All data reduction was carried out by the Cambridge Astronomical Survey Unit pipeline (http://www.ast.cam.ac.uk/ wfcsur/index.php). This included de-biasing, bad pixel replacement, non-linearity correction, flat-fielding, de-fringing, gain correction and photometric calibration.

\subsection{Data analysis}

The detection of LSB dwarf galaxies is subject to strong selection effects, due to their intrinsic low surface brightness that is, by definition, below the sky level (i.e. they consist of low signal-to-noise objects on a digital image). For this reason, standard algorithm based on a connected pixels above a threshold concept (e.g. SExtractor, Bertin\& Arnouts (1996)) are not ideal for their detection. The galaxy detection in this work was therefore perfomedded using a purposely built, fully automated, detection algorithm based on the convolution of the original images in the Fourier Space with a set of multi-scale top-hat filters. The method and its potentialities are discussed in Scaramella et al (this conf). The detection algorithm produces a list of candidate LSB galaxies that need to be selected against the background galaxies. In order to estimate the best selection criteria (i.e. the one that optimize cluster members detection and minimize background contamination of the sample), we run numerical simulations of a cone of Universe uniformly populated by galaxies and of a cluster at the position of the Virgo Cluster. The details of this detection algorithm and the selection criteria have been extensively discussed in Sabatini et al. (2003) and Roberts et al. (2004). The result of this process is that we selected galaxies that, at the distance of the Virgo cluster (16 Mpc, Jerjen et al (2004)), satisfy the following criteria: a central surface brightness of $23 \leqslant \mu_{o} \leqslant 26 \mathrm{~B} \mathrm{mag} / \mathrm{arcsec}^{2}$, and an exponential scale size $(h)$ in the range: $4 \leqslant h \leqslant 9$ arcsec. $\dagger$ Our final sample consist of 596 dwarf LSB galaxies (roughly $2 / 3$ out of which were previously uncatalogued) found over an area of $70 \mathrm{deg}^{2}$ in environments of increasing density.

$\dagger$ This actually corresponds to galaxies with intrinsic scale sizes of $3 \operatorname{arcsec}$ as it can be shown that convolution of the typical seeing function of our data with a 3 arcsec scale length resulted in a measured scale length of about 4 arcsec (Sabatini et al (2003)). 

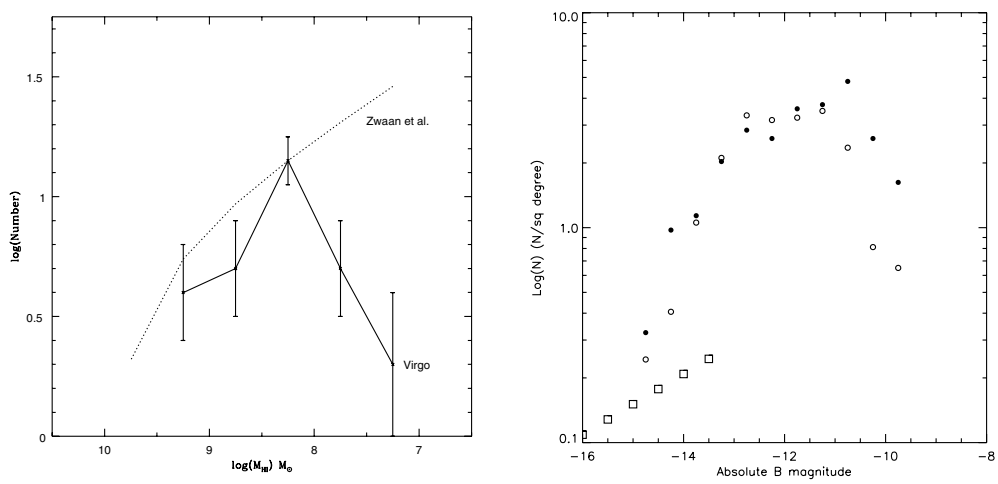

Figure 2. On the left: HI mass function. The solid line shows the mass function from the Virgo HIPASS data, the dashed line is the derived fit to the field galaxy mass function from Zwaan et al (2005), arbitrarily normalised to the peak on the Virgo data at $M_{H I}=8.5$. On the right: Virgo faint end part of the LF. Open circles are counts for the raw data and filled points are those corrected for detection efficiency and background contamination. Squares are points from the VCC LF with a slope of -1.3 .

\section{Results}

\subsection{Number density and Dwarf-to-Giant Ratio}

In the EW strip of the Virgo Cluster, we found on average about 18 galaxies per $\mathrm{deg}^{2}$ but ranging from about 40 per $\operatorname{deg}^{2}$ at the cluster centre to approximateley 4 per $\operatorname{deg}^{2}$ at the cluster edge (Sabatini et al (2003)). This decline in numbers with cluster radius is a good indicator that we have selected a predominately cluster population rather than background galaxies. In a related paper (Roberts et al (2004)) we compared the Virgo cluster result with field data from the MGS. Over this region the numbers of galaxies detected were only 4 per $\operatorname{deg}^{2}$, consistent with the numbers we detected at the edge of the Virgo cluster, again indicating that our cluster data consists predominately of cluster galaxies. Comparing the numbers of dwarf galaxies to that of the giant galaxy population over the same region of sky gave a Dwarf-to-Giant Ratio (DGR) of about $14\left(\mathrm{DGR}=\operatorname{Num}\left(M_{B} \leqslant-19\right) / \operatorname{Num}\left(-14 \leqslant M_{B} \leqslant-10\right)\right)$ for the Virgo E-W strip and 22 for the N-S strip. The DGR of the MGS strip was at most 6, consistent with the numbers of dwarf and giant galaxies found in the Local Group (Mateo, 1998) if we imagine to move the Local Group to the Virgo Cluster distance and apply the above discussed selection criteria. The Virgo cluster clearly supports many more dwarf galaxies per giant than do galaxies in less rich environments. Values of DGR are shown in table 1 (column 3) for the environments where it was possible to calculate them. Average number density of dwarf galaxies is also shown (column 2) and can be compared in different environments. The MGS survey clearly shows that there is no large field population of LSB low luminosity galaxies that would steepen the luminosity functions of Blanton et al (2001) and Norberg et al (2002) at fainter magnitudes, to account for the discrepancy with theoretical predictions. In the special environment of the Virgo cluster, consistently with previous shallower surveys, we found relatively large numbers of dwarf galaxies, that provide a little closer match to the theory (Moore et al., 1999).

\subsection{Properties of the dwarf galaxies in our sample}

In Sabatini et al (2005) we followed up the galaxies of our catalogue and analysed the B-I colours and gas content (thourgh observations of our candidates carried out at Arecibo, 
Table 1. Comparison of dwarf galaxy's properties in different environments

\begin{tabular}{llllllll}
\hline Region & $\begin{array}{l}<\text { Density } \\
\left(\mathrm{N} / \mathrm{deg}^{2}\right)\end{array}$ & $D G R$ & $\langle B-I\rangle$ & $\begin{array}{l}H I \\
(\text { det. rate })\end{array}$ & $\% d E$ & $\% d I r r$ & $\%$ Other \\
\hline MGS & 4 & $<6$ & $1.2 \pm 0.3$ & $23 \%$ & $24 \%$ & $33 \%$ & $43 \%$ \\
M101* & 3.6 & - & - & - & $40 \%$ & $38 \%$ & $22 \%$ \\
M101 & 0.1 & 1 & - & - & $100 \%$ & - & - \\
UMa & 4 & - & - & - & $33 \%$ & $17 \%$ & $50 \%$ \\
Virgo EW & 18 & 14 & $1.8 \pm 0.4$ & $5 \%$ & $62 \%$ & $26 \%$ & $12 \%$ \\
Virgo NS & 22 & 22 & $1.8 \pm 0.7$ & - & $54 \%$ & $22 \%$ & $24 \%$ \\
\hline
\end{tabular}

with a mass limit of $\sim 1.5 \times 10^{7} M_{\odot}$ at the distance of the Virgo Cluster and a column density limit of $\sim 5 \times 10^{18}$ atoms $/ \mathrm{cm}^{2}$ ). Table 1 shows average values of the B-I colours (column 4). Interestingly colours of the two different regions analysed in Virgo are similar, but the NS strip shows a higher dispersion: this could be due to the strip being populated of galaxies from further away infalling clouds (implying fainter magnitudes and therefore larger errors in the colour $\dagger$. On average dwarfs in Virgo are bluer than typical giant ellipticals in the cluster $((\mathrm{B}-\mathrm{I})=2.1 \pm 0.1$, Michard $(2000))$ and wihin the errors have similar (B-I) colour to typical globular clusters (GC) around M87 ((B-I) $=1.65 \pm 0.25$, Conture et al (1990)). It's also worth noticing that (B-I) colours of galaxies in the 2 cluster strips does not depend upon distance from the cluster centre.

HI detection rate for a subsample of the galaxies in our surveys of the Virgo Cluster and the field that were followed up are shown in table 1 (column 5): galaxies in the cluster tend to be gas poor compared with those in the field. In aggrement with this picture, although still under intense discussion at the low masses (see this conf), we find that the HI Mass Function (HIMF) in the cluster is shallower than in the field (see Fig 2). This has an opposite trend to the LF, that we find steep in Virgo and flat in the field (Fig 2), suggesting that the cluster plays an important role in promoting star formation and therefore in converting atomic gas into stars.

To summarise: in the comparison of Virgo cluster dwarf galaxies to MGS field dwarf galaxies, consistently with previous observations, we find that the cluster population is redder and gas poor.

Concerning the morphology of galaxies in the different environments analysed, again consistenly with previous results in the literature, we find that $\mathrm{dE}$ are more abundant in denser environments and tend to be a small percentage in the field (table 1, columns 6 to 8$)$.

\section{Discussion}

In what follows we will discuss the properties of these extremely faint low surface brightness galaxies as related to the environment in which they reside and evaluate which of the analysed physical mechanisms can be the main driver for their evolution. The main issue that we are trying to assess is wheter these galaxies are fossils of the primordial fluctuations predicted by $\Lambda \mathrm{CDM}$ models or they have been created lately. Kauffman, White \& Guiderdoni (1993) suggest that the missing DM halos must remain dark and so undetectable. If this is so, then a much larger fraction of field DM halos

$\dagger$ This picture is also supported by the comparison of the total magnitude distributions of the two sample and the surface brightness (SB) distributions: galaxies in the NS strip have fainter total apparent magnitude and than galaxies in the EW strip similar SB, suggesting that they are at a higher distance (Roberts et al (2007)) 
remain dark compared to those in clusters. The cluster environment must have played an important role in making these galaxies visible. Alternately if these dark halos do not survive to the present epoch, the cluster environment must have created the excess of dwarf galaxies compared to the field. In what follows we analyse mechanisms in favor of one or the other scenario.

\subsection{Creation of dwarfs}

- Harassment Moore et al (1999) showed that infalling LSB disk galaxies in a cluster can be morphologically transformed into dEs by the several high speed tidal interactions with giant cluster galaxies. We tested 2 predictions of this model:

1) size of the dEs in our sample. Moore et al. (1998) give the smallest radius of an harassed galaxy to be $\sim 1.67 \mathrm{Kpc}$, even if Sabatini et al (2005) calculated that if the dwarf galaxies found in the cluster originally came from a population of larger field galaxies then they should have tidal radii of the order $\sim 7 \mathrm{kpc}\left(r_{t} \simeq R_{c}\right.$ ore $\left.\sigma_{\text {gal }} / \sigma_{\text {clus }}\right)$. The dwarf galaxies in our sample have scale-lengths between 4 arcsec and $9 \operatorname{arcsec}$, which, at the distance of Virgo correspond to physical scale-sizes of $0.25 \mathrm{kpc}$ to $0.75 \mathrm{kpc}$, therefore smaller than the predicted sizes.

2) tidal streams (i.e. stars torn out from the harassed galaxies will lie along narrow streams which follow the orbital path of the galaxy). Davies et al 2005 carried out a search for these tidal arcs around $38 \mathrm{dE}$ galaxies found in our survey of the N-S strip, reaching the limit of $\sim 29 \mathrm{~B} \mathrm{mag} / \mathrm{arcsec}^{2}$ (which is $1.5 \mathrm{mag}$ fainter than the predicted surface brightness values for the stream stripped by an infalling $M_{B}=20$ galaxy distributed around a radius of $1 \mathrm{Mpc}$ and $2 \mathrm{Kpc}$ thick, Moore et al (1996)). From this search, Davies et al. found no evidence for tidal streams which could be associated with the sample of dEs.

A recent paper by Mastropietro et al (2005) shows that the size of harassed dEs could be smaller and streams surface brightness fainter than what originally predicted. However, the most problematic part of the harassment idea is where the initial LSB disk galaxies come from. As galaxy groups come together to form clusters this population should reside in the groups, while large numbers of LSB disc galaxies are just not found in galaxy groups.

- Tidal Dwarfs Slow speed close tidal interactions between galaxies in clusters result in gas and stars being pulled out from the interacting galaxies into giant streams, along which clumps of gas and stars form. Over time the stream fades, and the clump is classified as a Tidal Dwarf Galaxy (TDG). There is observational evidence of the extistence of this kind of galaxies (see e.g. Hunsberger et al (1996)) and simulations of the formation of TDGs predict that 1-2 of them form with each interaction (Okazaki \& Taniguchi (2000)). We can estimate how many TDG producing interactions there could be in Virgo by considering a simple rate equation. The number of interactions $(\mathrm{N})$ which may produce a TDG in a cluster depends upon four parameters - the number density of galaxies $(\rho)$, their interaction cross section $(\sigma)$, their velocity $(\mathrm{v})$ and the age of the cluster $(\mathrm{T})$. Thus,

$$
N \sim \rho \sigma v T
$$

If we assume that only interactions between disk galaxies ( $\mathrm{S} 0$ and spirals) produce TDGs (Okazaki \& Taniguchi (2000)), then using the information in Tully et al (1996) and assuming that the interaction cross-section is the virial radius, we estimate there to be about 13 interactions per Gyr. So, if we assume that each interaction makes, at most, 2 TDGs, this means that we would expect 26 TDGs to be formed every Gyr. It seems therefore extremely unlikely that TDGs make up a large fraction of the cluster 
dwarf galaxy population, and certainly not large enough to account for the dwarf galaxy population we find in Virgo today.

- Ram pressure stripping Galaxies infalling in a cluster with a hot intra-cluster medium are subject to its ram pressure that can be capable of stripping thier gas away. dIrrs could be particularly fragile with respect to this mechanism (van Zee et al (2004)) and could potentially be transformed to dEs. However, the importance of ram pressure stripping on the evolution of cluster dwarf galaxies in Virgo was investigated by Sabatini et al (2005) who found that, due to the expected high M/L ratio of these galaxies, only those dwarfs within the cluster core $\left(\sim 0.5 \mathrm{Mpc}\right.$ or $\left.1.5^{\circ}\right)$ would be affected by this process. For the E-W strip, they also conclude that the dwarfs they detect within the projected cluster core would be severely tidally disrupted if they were actually located in the core, thus they must be outside the core region, and therefore will not be subject to ram pressure stripping. The majority $(99 \%)$ of the galaxies detected in our N-S Virgo cluster strip are outside the projected core region due to the offset of this strip from the cluster centre. Thus the effect of ram pressure stripping on these galaxies must be small.

In Sabatini et al (2005) we suggest that enhanced star formation triggered by interactions with the cluster and galaxy potentials, accelerates the evolution of infalling DM halos so that they resemble the dEs which we see in Virgo today, a process that does not happen in the field. The (B-I) colour of our detections (discussed in section 3) are consistent with a stellar population that is younger than the giant elliptical galaxies.

\subsection{Suppression of dwarfs}

Having discussed the possible mechanisms which could create the large population of dwarf galaxies that we find in our survey of the Virgo cluster region, we now investigate the possible reasons why we detect very few dwarf galaxies in lower density environments (the general field, the region around M101 and the low density Ursa Major cluster).

- Supernovae winds In this scenario (Dekel \& Silk (1986)), the most commonly invoked when attempting to suppress the formation of dwarf galaxies, the first generation of SN injects enough energy into the halo gas, for it to escape the halo and thereby prevent further star formation, rendering the halo invisible. Babul \& Rees (1992) have suggested that this mechanism for gas expulsion may be environmentally dependent because the pressure of the intra-cluster gas will reduce gas loss in clusters (pressure confinement). However, it is not clear how efficient this mechanism for gas loss will be in any environment: MacLow \& Ferrara (1999) have investigated this idea using numerical simulations and showed that SN winds are effective at blowing out the gas only for haloes of masses $<10^{6} M_{\odot}$. Sabatini et al (2005) have also questioned the viability of this gas loss mechanism in the light of the very high mass-to-light ratios that have been derived for some dwarf galaxies. Note also that, using ASCA X-ray survey temperature maps, Roberts (2005) showed that galaxies in Virgo are pressure confined only within 3 deg of M87 in the NS strip and $2 \mathrm{deg}$ in the EW. Thus gas expulsion via SN winds does not appear to be able to explain why we see small numbers of dwarf galaxies only in low density environments.

- Re-ionization Are there many DM haloes present in low density environments that are not observable because they have not been lit up by star formation? One explanation for this is the presence of a photoionizing background preventing the gas in the halo from cooling. To account for an environmental dependence of this mechanism, we should refer to the so called 'squelching' scenario (Tully et al (2002)): in this picture high density cluster sized regions (like the Virgo Cluster) are assumed to form before the epoch of re-ionization (thus star formation in their dwarfs is not inhibited). Lower density regions 
(such as Ursa Major and the field) form later and thus the UV background heats the gas in the dwarfs residing in them, preventing it from cooling and forming stars. However, in their model, Tully et al (2002) used $z_{\text {reion }}$ of 6 , while the third year of WMAP results have pushed the epoch of re-ionization to $z=9 \pm 3$, a time when the formation of dwarf galaxy sized objects is rare.

Although the squelching scenario may have problems explaining the environmental dependence of dwarf galaxy populations, the effect of photoionization on low mass DM haloes may well play a part in the formation of galaxies in the idea known as 'downsizing' (Cowie et al (1996)). This scenario, born out of observational evidence that larger galaxies have older stellar populations than lower mass ones is at first sight contrary to hierarchical theory of structure formation. However, it is not contrary if for some reason star formation in low mass halos is in some way delayed, possibly delayed so long that large numbers of small halos have not yet undergone any star formation at all.

If photoionization does result in there being many low mass DM haloes in the Universe which have not been able to form stars to make them visible as dwarf galaxies, then gravitational lensing could be used as a probe of substructure. This is an ideal tool to use since light is deflected gravitationally by matter, whether it is light or dark, thus if there were small dark haloes present in the Universe, they could be detected by this means. Such studies have been carried out (Metcalf \& Zhao (2002), 2002, Bradac et al (2002), Dalal \& Kochanek (2002)) and preliminary results show evidence for the presence of substructure. Kochanek \& Dalal (2003) also rule out the possibilities of other effects causing the flux anomalies in a further study of their data, concluding that 'low mass haloes remain the best explanation of the phenomenon'. However, if these low mass DM haloes do exist in the numbers predicted by $\Lambda \mathrm{CDM}$, then as they fall through the disk of their parent galaxy, they should heat the disk and cause it to thicken (Tóht \& Ostriker (1992)). This is contrary to some observations of old thin disk systems or galaxies with no thick disk components, although it is now being argued that the amount of heating and thickening has been overestimated (Font et al (2001), Velázquez \& White (1999)). This is clearly a matter for further investigation.

\section{Conclusions}

Our suggested solution to the origin of the differences in the number counts and properties of dwarf galaxies in different environments is that there must be many very LSB or totally dark galaxies in the Universe that we have not yet been discovered. In the cluster environment many of these have been 'lit up' by enhanced star formation due to them being pulled and pushed around within the cluster environment. In support of this we cite the following results from our survey:

(a) The dE galaxies in Virgo are bluer than the giant ellipticals - their star formation was delayed until the cluster was formed.

(b) Dwarf galaxies in Virgo are redder and gas poor compared to those in the field the cluster environment promotes the conversion of gas into stars. Note that downsizing implies low efficiency star formation in the lowest mass objects - but more rapid in clusters?

(c) The galaxies we detect in Virgo are too small to be the result of harassment.

(d) There are too few tidal interactions in Virgo for them to be created tidally.

(e) There is a clear lack of dwarf galaxies in the dynamically young Ursa Major cluster.

(f) If the dwarfs have high mass-to-light ratios they will not be subject to gas lose by SN driven winds. 
Multi-band follow ups to try and constrain the stellar population, star formation history, age, metallicity of the dwarfs of our catalogue are necessary in order to better investigate their nature. We are currently using ugriz SDSS imaging data at this purpose for a subsample of them and we hope to obtain NIR data for constraining their stellar population.

The recent discovery of extremely low luminosity and low surface brightness dSph companions to the MW (Irwin et al (2007) and references therein) has highlighted the possibility that the predicted population of low mass haloes in $\Lambda$ CDM may actually exist. Kleyna et al (2005) comment that this new dSph, which has a M/L of over $500 \mathrm{M}_{\odot} / L_{\odot}$ and absolute magnitude, $M_{V} \sim-6.75$, "may represent the best candidate for a "missing" $\Lambda C D M$ halo'. They conclude that there must be more dark and massive dwarfs hiding in the region around the Milky Way. It is therefore extremely important that searches for such objects are carried out if we are to properly check the consistency of observations with $\Lambda$ CDM predictions.

\section{References}

Babul, A. \& Rees,M., 1992, MNRAS, 255, 346

Bertin, E., Arnouts, A., 1996, A \& A, 117, 393

Binggeli, B., Sandage, A., Tarenghi, M., 1984, AJ, 89, 64

Blanton, M.R., Dalcanton, J., Eisenstein, D., Loveday, J., Strauss, M., SubbaRao, M., Weinberg, D.H., Anderson, J.E. Jr. and 61 coauthors,2001, AJ,121, 2358

Blanton, M.R., Eisenstein, D., Hogg, D.W., Schlegel, D.J. \& Brinkmann, J.,2005, ApJ, 631, 208

Bradac, M., Schneider, P., Steinmetz, M., Lombardi, M., King, L. \& Porcas, R., 2002, AA, 388, 373

Conture, J., Harris, W. \& Allwright, J., 1990, ApJS, 73, 671

Cowie, L., Songaila, A., Hu, E. \& Cohen, J., 1996, AJ, 112, 839

Dalal, N. \& Kochanek C., 2002, ApJ, 572, 25

Davies, J.I., Roberts, S. \& Sabatini, S., 2005, MNRAS, 356, 794

Dekel, A. \& Silk, J., 1986, textitApJ, 303, 39

Font, A., Navarro, J., Stadel, J. \& Quinn, T., 2001, ApJ, 563, L1

Hunsberger, S., Charlton, J. \& Zaritsky, D., 1996, ApJ, 462, 50

Irwin, M.J., Belokurov, V., Evans, N.W., Ryan-Weber, E.V., de Jong, J.T.A., Koposov, S., Zucker, D.B., Hodgkin, S.T. and 19 coauthors,,2007, ApJ, 656L, 13

Jerjen, H., Binggeli, B., Barazza, F., 2004, AJ, 127, 771

Kambas, A., Davies, J.I., Smith, R., Bianchi, S., Haynes J.A.,2000, AJ, 120, 1316

Kauffman, G., White S.D.M. \& Guiderdoni B., 1993, MNRAS, 264, 201

Kleyna, J., Wilkinson, M.I., Evans, N.W., Gilmore, G., Frayn, C., 2005, ApJ, 630, 141

Klypin, A., Kravtsov, A.V., Valenzuela O. \& Prada, F., 1999, ApJ, 522, 82

Kochanek, C. \& Dalal, N., 2003, AIP Conf. Proc. 666, 'The Emergence of Cosmic Structure', p. 103

Liske, J., Lemon, D., Driver, S., Cross, N. \& Couch, W., 2003, MNRAS, 344, 307

MacLow, M. \& Ferrara, A., 1999, ApJ, 513, 142

Mastropietro, C., Moore, B., Mayer, L., Debattista, V.P., Piffaretti, R., Stadel, J.,, 2005, MN$R A S, 364,607$

Metcalf, R. \& Zhao, H., 2002, ApJ, 567, L5

Michard, R., 2000, AA, 360, 85

Moore, B., Katz, N., Lake, G., Dressler, A. \& Olmer, A.,1996, Nature, 379, 613

Moore, B., Ghigna, S., Governato, F., Lake, G., Quinn, T., Stadel, J. \& Tozzi, P., 1999, ApJ, 524, L19

Norberg, P., Cole, S., Baugh, C.M., Frenk, C.S., Baldry, I., Bland-Hawthorn, J., Bridges, T., Cannon, R., and 20 coauthors,2002, textitMNRAS, 336, 907

Okazaki, T. \& Taniguchi, Y., 2000, ApJ, 543, 149 
Roberts, S., Davies, J.I., Sabatini, S., van Driel, W., O’Neil, K., Baes, M., Linder, S.M., Smith, R., Evans, R., 2004, MNRAS, 352, 478

Roberts, S., 2005, PhD Thesis, Cardiff University

Roberts, S., Davies, J.I., Sabatini, S., Auld, R., Smith, R.,2007 MNRAS, in press

Sabatini, S., Davies, J.I., Scaramella, R., Smith, R., Baes, M., Linder, S.M., Roberts S. \& Testa, V., 2003, MNRAS, 341, 981

Sabatini, S., Davies, J.I., Van Driel, W., Baes, M., Robert, S., Smith, R., Linder, S.,O’Neil, K., 2005, MNRAS, 357, 819

Simon, J.D. \& Geha M., 2007, submitted to ApJ (preprint: astro-ph/0706.0516v1)

Toth, G. \& Ostriker, J., 1992, ApJ, 389, 5

Tully, B.R., Verheijen, M.A.W., Pierce, M.J., Huang, J.S., Wainscoat, R.J.,1996, AJ, 112, 2471

Tully, B.R., Somerville, R., Trentham, N. \& Verheijen, M., 2002, ApJ, 569, 573

van Zee, L., Barton, E.J. \& Skillman, E.D., 2004, AJ, 128, 2797

Velázquez, H. \& White, S., 1999, MNRAS, 304, 254

Zwaan, M.A., Stavely-Smith, L., Koribalski, B.S., Henning, P.A., Kilborn, V.A., Ryder,, S.D., Barnes, D.G., Bhathal Rl et al, 2005, AJ, 1255, 2842

\section{Discussion}

CORTESE: I am wondering if in comparing the contribution of dwarf galaxies in different environments you have considered that the field is underdense, i.e. the number of galaxies in the field is small and therefore relative errors on counts are large. Can the errors take into account of the discrepancy and reconcile the faint end slope of the Virgo Cluster and that of the Field? Same comment on the DGR.

SABATINI: Number counts in the faint part of the LF in the field indeed were so low that we couldn't do a LF. This is why we have used the DGR for the comparison - the DGR allows to rescale taking into account the different density of the environments. However, indeed relative errors in counts are larger in the field, but I don't think they could be responsible for reconciling the discrepancy: we would have to have missed an order of magnitude in number of dwarfs in the field compared to the centre of the cluster.

Moore: What about Valotto et al 2001?

SABATINI: Valotto et al show that using statistical subtraction for the estimation of the background contamination, can lead to artificially steep LFs. For this reason, we do not use control fields for a statistical estimation of the background. We instead run numerical simulations to model the background galaxy population and find the best selection criteria (based on morphology) that allows to minimize the background contamination. A far away galaxy looks faint and could in principle be a contaminant in the cluster LF, but selecting galaxies for their scale length and surface brightness ensures us to get rid of these contaminants, that would appear smaller and with higher surface brightness at a given magnitude if compared to local LSB galaxies. 